BULLETIN OF THE

AMERICAN MATHEMATICAL SOCIETY

Volume 80, Number 3, May 1974

\title{
ON QUOTIENTS OF MANIFOLDS: \\ A GeNeralization OF THE CLOSED SUBGROUP THEOREM
}

\author{
BY HÉCTOR J. SUSSMANN ${ }^{1}$
}

Communicated by Glen E. Bredon, October 10, 1973

Let $M$ be a ( $C^{\infty}$, Hausdorff, paracompact) manifold, and let $R$ be an equivalence relation on $M$. Then $R$ is called regular if the quotient $M / R$ is a (not necessarily Hausdorff) manifold in such a way that the canonical projection $\pi_{R}: M \rightarrow M / R$ is a submersion. For results on regular relations cf. Palais [1], Serre [2]. The following characterization of regularity is wellknown (cf. Serre [2, LG, Chapter 3, §12]): $R$ is regular if and only if it is a submanifold (with the subspace topology) of $M \times M$ in such a way that the map $\left(m, m^{\prime}\right) \rightarrow m$ from $R$ onto $M$ is a submersion.

The purpose of this note is to announce a different characterization of regularity. Proofs will appear elsewhere (Sussmann [3]).

Our condition is motivated in a natural way by Systems Theory. As will be shown in [4], Theorem 2 is precisely what is needed to show that, under fairly general conditions, every finite-dimensional "controllable" nonlinear system has a realization which is both "controllable" and observable.

Here we shall not pursue this line. Rather, we shall state our condition and show that it is a rather natural generalization of the closed subgroup theorem.

Let $X$ be a vector field on an open subset of $M$. We say that $X$ is a symmetry vector field of $R$ if, whenever $\left(m, m^{\prime}\right) \in R$, it follows that $\left(X_{t}(m), X_{t}\left(m^{\prime}\right)\right) \in R$ for every real $t$ for which $X_{t}(m)$ and $X_{t}\left(m^{\prime}\right)$ are both defined (here $t \rightarrow X_{t}(m)$ is the integral curve of $X$ which passes through $m$ when $t=0)$. Let $S^{\infty}(R, M)$ denote the set of all $C^{\infty}$ vector fields $X$ defined on open subsets of $M$ that are symmetry vector fields for $R$. It is not difficult to show that $S^{\infty}(R, M)$ is a presheaf of Lie algebras of vector fields. If $L$ is a set of vector fields defined on open subsets of $M$, we say that $L$ is transitive if, for every $m \in M$, the vectors $X(m), X \in L$, span the tangent space of $M$ at $m$. If $A$ is a subset of $M \times M$, we call $L A$-transitive if, for every $\left(m, m^{\prime}\right) \in A$, the tangent space of $M$ at $m$ is spanned by the

AMS (MOS) subject classifications (1970). Primary 58A05.

${ }^{1}$ Work partially supported by NSF Grant No. GP-37488.

Copyright (C) American Mathematical Society 1974 
vectors $X(m)$, where $X \in L$ is a vector field whose domain of definition contains both $m$ and $m^{\prime}$.

The equivalence relation $R$ is called locally regular if every $m \in M$ has a neighbourhood $U$ such that $R \cap(U \times U)$ is a regular equivalence relation in $U$. Similarly, we call $R$ locally closed if every $m \in M$ has a neighborhood $U$ such that $R \cap(U \times U)$ is closed in $U \times U$.

THEOREM 1. $R$ is locally regular if and only if it is locally closed and $S^{\infty}(R, M)$ is transitive.

THEOREM 2. Let $M$ be connected. Then $R$ is regular if and only if it is locally closed and $S^{\infty}(R, M)$ is $R$-transitive.

REMARK. As is well known, if $R$ is regular, then $M / R$ is Hausdorff if and only if $R$ is closed.

When $M$ is not connected, the condition of Theorem 2 is sufficient for regularity, except for the fact that the connected components of $M / R$ will not necessarily have the same dimension. Let us call $R$ almost regular if every connected component $M^{\prime}$ of $M / R$ can be given a differentiable structure in such a way that the canonical projection from $\pi_{R}^{-1}\left(M^{\prime}\right)$ onto $M^{\prime}$ is a submersion. Then Theorem 2 has an analogue that does not require $M$ to be connected.

THEOREM 2'. Let $M$ be an arbitrary $C^{\infty}$ manifold. Then $R$ is almost regular if and only if it is locally closed and $S^{\infty}(R, M)$ is $R$-transitive.

The preceding theorems have been stated in the $C^{\infty}$ category, but they are equally valid in the real analytic case (for instance, if $M$ is a connected real analytic manifold, and if the set $S^{\omega}(R, M)$ of real analytic symmetry vector fields of $R$ is $R$-transitive, then $M / R$ is a real analytic manifold in such a way that $\pi_{R}: M \rightarrow M / R$ is a real analytic submersion). The following two theorems, however, are stated for the $C^{\infty}$ case, and our proofs depend on arguments that have no real analytic analogue (such as the use of $C^{\infty}$ functions with compact support). We do not know whether the "only if" parts of Theorems 3 and 4 are also valid in the real analytic case (but it is easy to see that the "if" parts are).

THEOREM 3. Let $M$ be a connected $C^{\infty}$ manifold. Then the equivalence relation $R$ is regular if and only if $R$ is locally closed and the set of everywhere defined symmetry vector fields of $R$ is transitive.

A Theorem $3^{\prime}$ (in which $M$ is not required to be connected and regularity is replaced by almost regularity) is also valid.

Finally, the following characterizes those relations $R$ for which $\pi_{R}$ is a fibre map. 
THEOREM 4. Let $M$ be a connected $C^{\infty}$ manifold and let $R$ be an equivalence relation on $M$. Then $R$ is regular and the projection $\pi_{R}$ is a fibre map, if and only if $R$ is locally closed and there exists a transitive set of complete everywhere defined symmetry vector fields of $R$.

Again, there is an analogous theorem for the nonconnected case, in which regularity is replaced by almost regularity.

We remark that Theorem 4 can be applied in particular if $M$ is a Lie group and if $R$ is the equivalence relation induced by a closed subgroup $H$. The transitive set of symmetry vector fields is, simply, the Lie algebra of $M$. Therefore Theorem 4 implies that $R$ is regular (so that $H$ is a regular submanifold of $M$ ) and that $G / H$ is a manifold in such a way that the projection $g \rightarrow g H$ is a fibre map. This shows that the closed subgroup theorem is contained on our results.

REMARK. Another particular case of Theorem 4 is Ehresman's theorem on proper submersions.

\section{BIBLIOGRAPHY}

1. R. Palais, A global formulation of the Lie theory of transformation groups, Mem. Amer. Math. Soc. No. 22 (1957). MR 22 \#12162.

2. J. P. Serre, Lie algebras and Lie groups, Lectures given at Harvard University, 1964, Benjamin, New York, 1965. MR 36 \#1582.

3. H. J. Sussmann, A generalization of the closed subgroup theorem to quotients of arbitrary manifolds J. Differential Geometry (to appear).

4. - Observable realizations of nonlinear systems (submitted).

Department of Mathematics, Rutgers University, New Brunswick, New JERSEY 08903 